\title{
Comparative Evaluation of Root Canal Configuration and Root Canal Curvatures of Mandibular Premolars in South Indian Population - A CBCT Based Analysis
}

\author{
Anisha.A.Mahtani ${ }^{1}$ and S.Pradeep ${ }^{2}$ \\ ${ }^{1}$ Saveetha Dental College and Hospitals Saveetha Institute of Medical \\ and Technical Sciences Saveetha University,Chennai-77, India \\ ${ }^{2}$ Reader Department of Conservative Dentistry and Endodontics, Saveetha Dental College and Hospitals \\ Saveetha Institute of Medical and Technical Sciences Saveetha University ,Chennai-77, India
}

\section{ABSTRACT}

Several studies have stated the root canal morphology of premolars to be unique. The purpose of this article is to evaluate the root canal configuration and curvatures of mandibular premolars using Cone beam computed tomography amongst genders. This would enable us to understand the morphology and reduce the chances of failure of root canal treatments. 25 CBCT images (100 teeth) including those of mandibular premolars of 12 male (48 teeth) and 13 female (52 teeth) patients were selected. The images were observed with Galileos Viewer Sirona 3D. The images were used to evaluate the number of roots and root canals, root canal morphology according to Vertucci's classification and the direction of root apex curvature. According to this study, Type I Vertucci canal configuration was most prevalent in both mandibular premolars. Distal canal curvature was more in males and distal and lingual curvatures were found to be more in females in mandibular first premolars. Distal canal curvature was found to be more across both the genders while labial canal curvature was absent in both genders in mandibular second premolars. There was no significant association between men and women in root canal configurations or root canal curvatures ( $p>0.05)$.Identification of root canal morphology of complex mandibular premolars would reduce the chances of root canal failures by making sure all canals are obturated. Identification of direction of root canal curvature is essential to pre-curve the files prior to negotiation of canals to prevent perforation. The most prevalent root canal type in both premolars was Type I Vertucci and the most prevalent root canal curvature was distal

KEY WORDS: CBCT, PREMOLARS, ROOT CANAL MORPHOLOGY, ROOT CANAL CURVATURE, VERTUCCI'S CLASSIFICATION.

\section{ARTICLE INFORMATION}

${ }^{*}$ Corresponding Author: pandu.pradeep@gmail.com

Received 13th June 2020 Accepted after revision 9th August 2020

Print ISSN: 0974-6455 Online ISSN: 2321-4007 CODEN: BBRCBA

Thomson Reuters ISI Web of Science Clarivate Analytics USA and Crossref Indexed Journal

\section{Clarivate
Analytics}

NAAS Journal Score 2020 (4.31) SJIF: 2020 (7.728)

A Society of Science and Nature Publication,

Bhopal India 2020. All rights reserved.

Online Contents Available at: http//www.bbrc.in/

Doi: http://dx.doi.org/10.21786/bbrc/13.7/32 


\section{INTRODUCTION}

Mandibular premolars are known as an Enigma of Endodontics. They have one of the most complex anatomies. Treatment failures and post-treatment flareups have been majorly reported in these teeth, thus indicating the existence of too much variation in the root canal morphology. (Berman DDS and Hargreaves, 2015; Glassman, 1987) A thorough knowledge of root canal morphology is crucial for the success of root canal treatment (Vertucci, 2005). The internal root canal complexities are genetically categorized and they have a significant role in anthropology, hence emphasizing the identification of root canal morphologies of different ethnic populations (Neelakantan et al., 2010).

Weine, Vertucci, and Gulabivala (Gulabivala et al., 2001; Vertucci, 1984; Weine et al., 1969) classified and described the root canal system of human permanent teeth. Vertucci's classification is the most commonly used. The canal configurations were categorized into the following eight types based on Vertucci's classification (Vertucci, 1984).

Root canal configuration has been identified using different methods such as 2D radiography, root canal staining, hard tissue section and micro-CT but they miss the important third dimension which could lead to missing canals and buccolingual curves of root canals. (Alfawaz et al., 2019; Hajihassani et al., 2017) Cone-beam computed tomography scanning is a noninvasive 3D imaging technique which provides threedimensional high-resolution images with the possibility of removing the superimposed structures.(Yu et al., 2012) Tachibana and Matsumoto (1990) introduced it in the field endodontics (Tachibana and Matsumoto, 1990). It provides images of root morphology with more details than those obtained by conventional periapical radiography and will help in improving the endodontic treatment outcomes (Patel et al., 2007). In addition, it has a significantly lowered radiation dose compared to medical computed tomography (CT) (Suomalainen et al., 2009; Tsiklakis et al., 2005) and majority of the CBCT equipment are ergonomically designed for safe use and optimal performance(Llena et al., 2014)

We have numerous highly cited publications on well designed clinical trials and lab studies (Azeem and Sureshbabu, 2018; Govindaraju et al., 2017; Janani and Sandhya, 2019; Jenarthanan and Subbarao, 2018; Khandelwal and Palanivelu, 2019; Malli Sureshbabu et al., 2019; Manohar and Sharma, 2018; Nandakumar and Nasim, 2018; Poorni et al., 2019; Rajakeerthi and Ms, 2019; Rajendran et al., 2019; Ramarao and Sathyanarayanan, 2019; Siddique, Nivedhitha, et al., 2019; Siddique, Sureshbabu, et al., 2019; Siddique and Nivedhitha, 2019; Teja et al., 2018). This has provided the right platforms for us to pursue the current study. The aim of the present study is to evaluate the root canal configuration and root curvatures of mandibular premolars using Cone beam computed tomography amongst genders.

\section{MATERIAL AND METHODS}

Study design: The present study was conducted in the Department of Conservative Dentistry and Endodontics at Saveetha Dental College, Chennai. This study was done with the use of 25 Cone Beam Computer Tomography images which contained 100 mandibular premolar teeth of patients attending the hospital for treatment. 12 were male ( 48 teeth) and 13 were female ( 52 teeth) patients. The study was initiated after approval from the institutional review board. The study was generalised to the South Indian population.

\section{Inclusion and Exclusion criteria}

CBCT images included in the study contained,

- teeth without periapical lesions

- non- endodontically treated teeth

- no open apex canals

- CBCT images of high quality

The exclusion criteria was missing or incomplete data. Cross verification of data for errors was done with the help of clinical photographs.

Data Collection: The CBCT images were observed with Galileo's Viewer Sirona 3D and were evaluated by 2 examiners. The images were used to evaluate:

- number of roots and root canals

- root canal morphology according to Vertucci's classification

- direction of root canal curvature

Figure 1: СBCT of 34 shows distally curved root canal

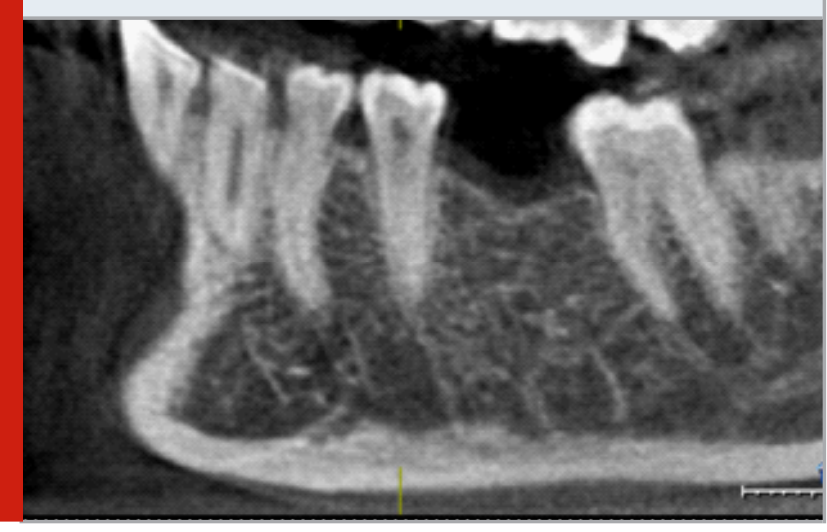

Radiographic technique and image analysis: The images for the study were selected from the radiographic database of the dental hospital. The images were acquired from a standard protocol for patient positioning, exposure parameter setting and image acquisition. The images were analysed on Galileo's Viewer Sirona 3D. The slice thickness of the CBCT is 150 micrometer.The variables evaluated were the number of roots and root canals, direction of root canal curvature and root canal morphology according to Vertucci's classification. The direction of root canal curvatures were divided into 4 categories:Mesial, Distal, Labial, Lingual. CBCT images 
of the curvatures are represented in Figure 1,2. The root canal configurations were evaluated based on the 8 types in Vertucci's classification (Vertucci, 1984).

Figure 2: CBCT of 35 shows lingually curved root canal

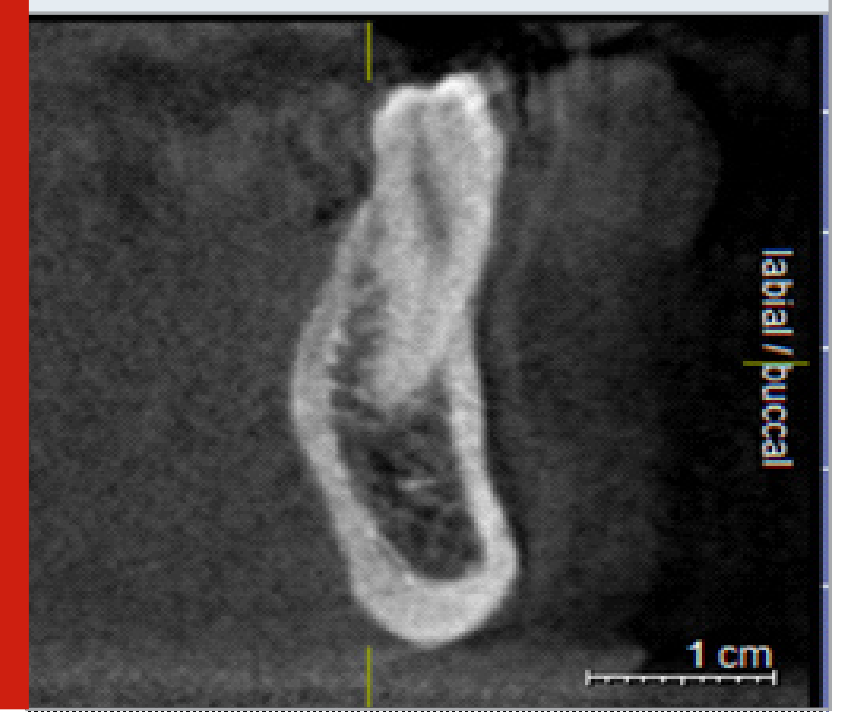

Statistical Analysis: The collected data was validated, tabulated and analysed with Statistical Package for Social Sciences for Windows, version 20.0 (SPSS Inc., Chicago, IL, USA). Percentage distribution of Vertucci's root canal configurations and root apex curvatures of mandibular premolars were calculated. Pearson's chisquare association was used to test associations between the gender of patients with root canal configuration and gender of patients with root apex curvatures of mandibular first and second premolars; $p$ value $<0.05$ was considered statistically significant.

\section{RESULTS AND DISCUSSION}

Since mandibular premolars have complex anatomies, studies regarding their anatomical variations could lead to better knowledge prior to root canal treatment. The aim of the present study is to evaluate the root canal configuration and root apex curvatures of first and second mandibular premolars using Cone beam computed tomography amongst genders.

On comparison of Vertucci's root canal configuration among genders, in mandibular first premolars, Type 1 configuration was seen in $40 \%$ of males and $36 \%$ of females, Type 2 configuration was seen in $6 \%$ of males and $8 \%$ of females and Type 5 configuration of root canals was seen in 2\% and $8 \%$ of males and females respectively. Type I root canal configuration was found to be more across both the genders. Chi square test was performed to test the association of the root canal configuration of mandibular first premolars with gender. The association was found to be not significant. Pearson Chi-Square Value - 1.97; $p=0.373$ ( $p$ value $>0.05$ ); not statistically significant, proving gender of patients is not associated with the root canal configuration of the mandibular first premolars (Fig 3).
Figure 3: Bar graph depicting the association of gender of patients with Vertucci's root canal configurations of mandibular first premolar. $\mathrm{X}$ axis represents the gender of patients and $\mathrm{Y}$ axis represents the number of mandibular first premolars. Type I root canal configuration (Blue) was found to be more across both the genders. However, Chi square test was performed and the association was not significant. Pearson Chi-Square Value - 1.97; $p=0.373$ (p value $>0.05$ ); not statistically significant.

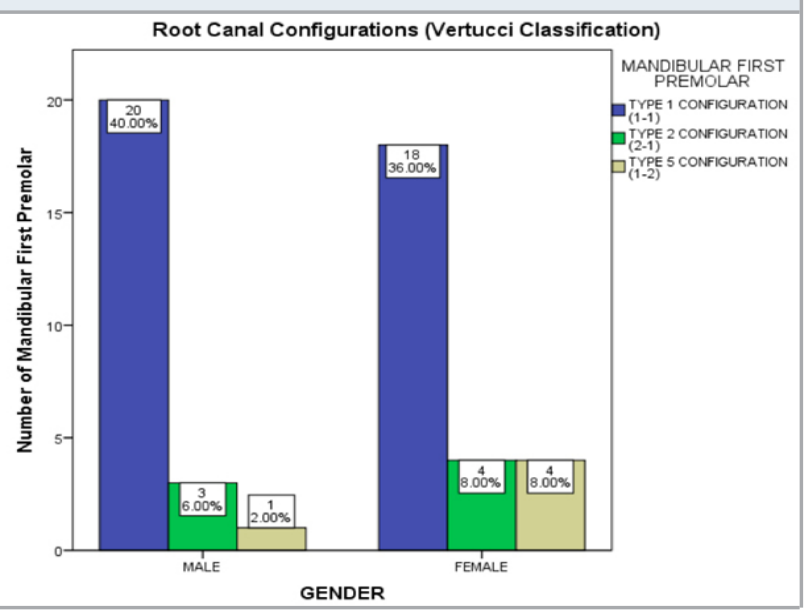

This statement was supported by a study conducted in the Iranian population (Kazemipoor et al., 2015), where the majority of mandibular first premolars (63.9\%) had Type 1 configuration ie. one canal and in which the association between the number of root canals in the mandibular first premolars in the two genders was statistically non significant $(P=0.081)$. However regarding the Vertucci classification in the mandibular first premolar in the Portugese subpopulation (Martins et al., 2018), the statement was opposed as a significant association was found in Type I (1-1) configuration with 72.7\% [68.4-77.0 CI 95\%] in males and 80.7\% [77.7-83.7 CI 95\%] in females. Huang et al (Huang et al., 2015) performed an analysis of the mandibular first premolar in Taiwan patients and concluded that females had a significantly higher number of singlerooted first premolars with one root canal, while males had a significantly higher prevalence of two-rooted first premolars with two root canals.

In mandibular second premolars, Type 1 configuration was seen in 36\% of males and 34\% of females, Type 2 configuration was seen in 10\% of males and $12 \%$ of females and Type 5 configuration of root canals was seen in $2 \%$ and $6 \%$ of males and females respectively. Type I root canal configuration was found to be more across both the genders. Chi square test was performed to test the association of the root canal configuration of mandibular second premolars with gender. The association was found to be not significant. Pearson Chi-Square Value - 1.04; $p=0.594$ ( $p$ value $>0.05$ ); not statistically significant, proving gender of patients is not associated with the root canal configuration of the mandibular second premolars (Fig 4). 
Figure 4: Bar graph depicting the association of gender of patients with Vertucci's root canal configurations of mandibular second premolar. $\mathrm{X}$ axis represents the gender of patients and $\mathrm{Y}$ axis represents the number of mandibular second premolars. Type I root canal configuration (Blue) was found to be more across both the genders. However, Chi square test was performed and the association was not significant. Pearson Chi-Square Value - 1.04; $\mathrm{p}=0.594$ (p value $>0.05$ ); not statistically significant.

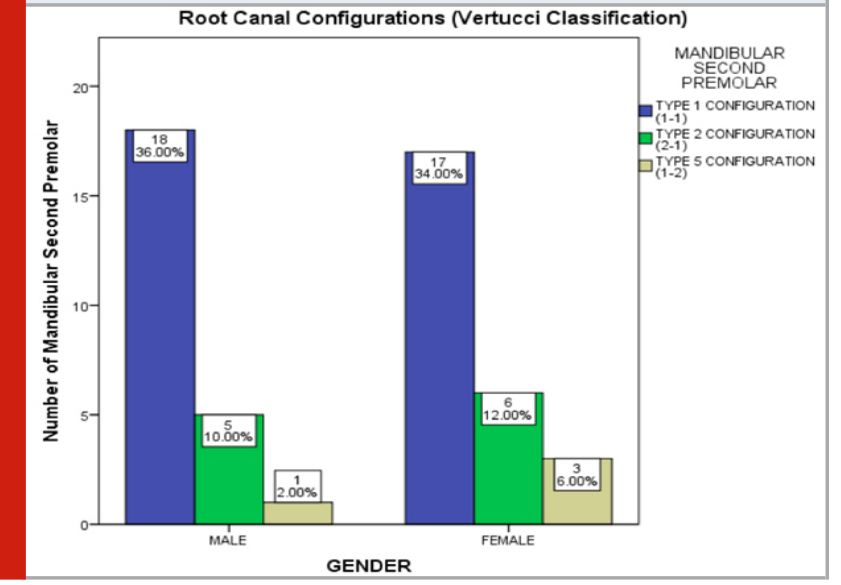

This statement was also supported by the study conducted in the Iranian population(Kazemipoor et al., 2015), where the majority of mandibular second premolars (78.3\%) had Type 1 configuration and there was no significant association between the two genders in the number of root canals $(P=0.498)$ in the mandibular second premolars. Of the reported studies, females have a higher likelihood of two or more roots or canals in mandibular first premolars, whereas men exhibit multiple canals much more frequently than females in mandibular second premolars (Iyer et al., 2006; Serman and Hasselgren, 1992; Sert and Bayirli, 2004). Others have reported no significant difference in root configuration between females and males (Miyoshi et al., 1974).

Specific types of canal morphology are visible in different racial groups. A higher incidence of two canals in mandibular first premolars was reported in several populations, upto 50\% in Indian populations (Velmurugan et al., 2010) and approximately 40\% in Middle Eastern populations from Kuwait(Zaatar et al., 1997) , Jordan (Awawdeh and Al-Qudah, 2008), and Turkey (Çaliskan et al., 1995). In the Malaysian population ((Kacharaju et al., 2019) 71\% of the single-canal premolars (Type 1) were observed in all three cross-sectional views. A study conducted by Lei Dou (Dou et al., 2017), in the Chinese population in mandibular first premolars, Type I canal system was found in 114 teeth (64.04\%), Types II, III, IV, V and VIII in 2 teeth (1.12\%), 19 teeth (10.67\%), 1 tooth $(0.56 \%), 39$ teeth $(21.91 \%)$, and 2 teeth $(1.12 \%)$ respectively and only one tooth $(0.56 \%)$ belonged to an additional type (1-3-1).

In the Jordanian population (Awawdeh and Al-Qudah, 2008) however, two separate apical foramina (Type IV) were found in 33\% of the teeth with two canals, compared to $6.2 \%$ with one apical foramen (Type I). Teeth with three separate apical foramina (Type VIII) were scarce (2.2\%), this statement supported the current study which found no three separately distinct root canals extending to the apex. Majority of the mandibular second premolars were found to have a single canal; $72 \%$ of the teeth possessed type I canal systems, whilst $22.8 \%$ of the roots had two canals with two separate apical foramina in the Jordanain population (Awawdeh and Al-Qudah, 2008).

In a systematic review conducted by Kottoor et al, mandibular first premolars were found to have one root $(97.21 \%)$ in most instances. However, only $73.55 \%$ of these single rooted teeth contained a single canal.The mandibular first premolar was more prone to bifurcation of canals (23-30\%) terminating in multiple apical foramina (15-20\%), as compared to second premolars. Type I canal configuration was most prevalent in both first (72.6\%) and second premolars (83.65\%) (Kottoor et al., 2013). In a study conducted by FJ Vertucci (Vertucci, 1978), the mandibular first premolar had one canal in $74 \%$ of the teeth, two canals in $25.5 \%$, and three canals in $0.5 \%$ of the teeth. The mandibular second premolar had $97.5 \%$ one canal at the apex and $2.5 \%$ two canals at the apex respectively.

Figure 5: Bar graph depicting the association of gender of patients with the root canal curvature of mandibular first premolar. $\mathrm{X}$ axis represents the gender of patients and $\mathrm{Y}$ axis represents the number of mandibular first premolars. Distal curvature (blue) was more in males, Distal (blue) and lingual (purple) curvatures were found to be more in females. However, Chi square test was performed and the association was not significant. Pearson Chi-Square Value $-0.72 ; p=0.868$ ( $p$ value $>0.05$ ); not statistically significant.

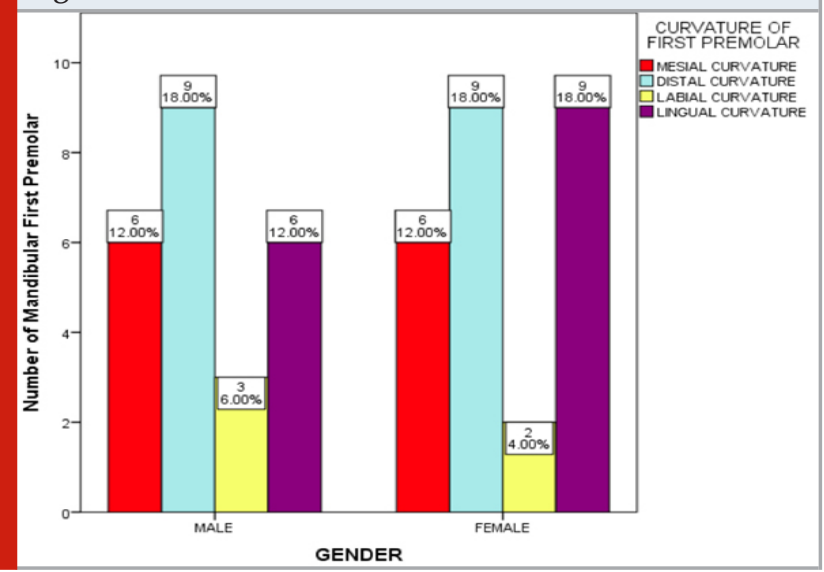

On comparison of the root canal curvatures among genders, in mandibular first premolars, mesial curvature was seen in $12 \%$ of males and females, distal curvature was seen in 18\% of males and females, labial curvature was seen in 6\% of males and $4 \%$ of females and lingual curvature was seen in $12 \%$ and $18 \%$ of males and females respectively. Distal curvature was more in males and distal and lingual curvatures were found 
to be more in females in mandibular first premolars. Chi square test was performed to test the association of the curvatures of root canals of mandibular first premolars with gender. The association was found to be not significant. Pearson Chi-Square Value - 0.72; p $=0.868$ ( $\mathrm{p}$ value $>0.05)$; not statistically significant, proving gender of patients is not associated with the root canal curvatures of the mandibular first premolars (Fig 5). No significant difference was also found between men and women $(p>0.05)$ in a study conducted by Hajihassani N(Hajihassani et al., 2017), thus supporting our statement.

In mandibular second premolars, mesial curvature was seen in 8\% of males and 10\% of females, distal curvature was seen in 22\% of males and $24 \%$ of females and lingual curvature was seen in 18\% of males and females. Distal canal curvature was found to be more across both the genders while labial canal curvature was absent in both genders in mandibular second premolars. Chi square test was performed to test the association of the curvatures of root canals of mandibular second premolars with gender. The association was found to be not significant. Pearson Chi-Square Value - 0.075; $p=0.963$ ( $p$ value $>0.05$ ); not statistically significant, proving gender of patients is not associated with the root canal curvatures of the mandibular second premolars (Fig 6). This was supported by Hajihassani N(Hajihassani et al., 2017) who also found no significant difference between men and women in his study ( $p>0.05)$.

Figure 6: Bar graph depicting the association of gender of patients with the root canal curvature of mandibular second premolar. $\mathrm{X}$ axis represents the gender of patients and $\mathrm{Y}$ axis represents the number of mandibular second premolars. Distal curvature (blue) was found to be more across both the genders while Labial curvature (yellow) was absent in both genders. However, Chi square test was performed and the association was not significant. Pearson Chi-Square Value - 0.075; $p=0.963$ ( $p$ value $>0.05$ ); not statistically significant.

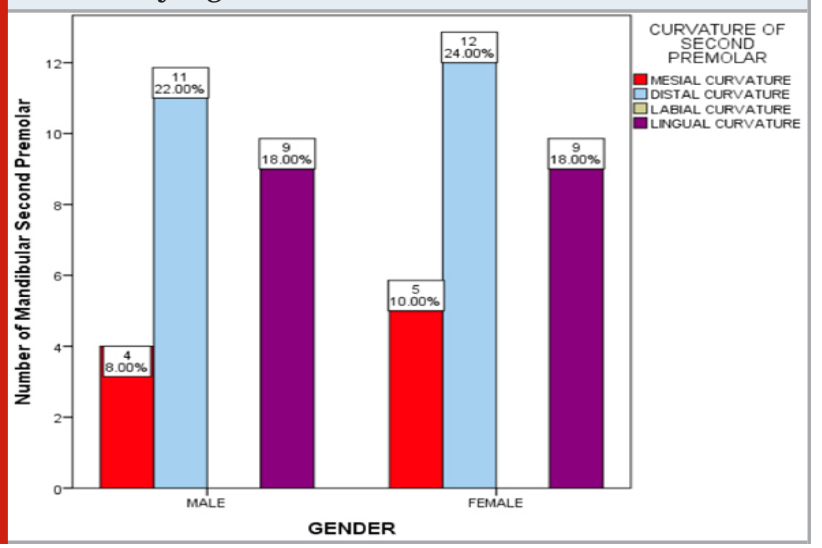

In mandibular first premolars, distal and lingual canal curvatures were most prevalent in females with $18 \%$ of occurrence while in males, distal curvatures were more prevalent (18\%). In mandibular second premolars as well distal curvatures were most prevalent in females (24\%) and males (22\%). This statement was supported by a study conducted in the Iranian population (Hajihassani et al., 2017) which stated the most prevalent canal curvature to be lingual for the first premolars (7.6\%) and distal for the second premolars (11\%). No significant difference was found between men and women ( $p>0.05)$. They also found that the most prevalent root curvature was the distal curvature in both premolars; with 71.4\% and 74\% of first and second premolars, respectively.

\section{CONCLUSION}

Identification of root canal morphology of complex mandibular premolars would reduce the chances of root canal failures by making sure all canals are obturated. Identification of direction of root canal curvature is essential to pre-curve the files prior to negotiation of canals to prevent perforation. The most prevalent root canal type in both premolars was Type I Vertucci and the most prevalent root canal curvature was distal.

\section{ACKNOWLEDGEMENTS}

We would like to thank the administration of Saveetha University, Chennai for granting us the clearance to conduct this study and for funding this research.

Authors Contribution: Pradeep.S contributed to study conception and design, data collection, analysis and interpretation and drafted the work. Anisha Mahtani contributed to data interpretation, study design and data collection. All authors critically reviewed the manuscript and approved the final version.

Conflict of Interest: The authors declare no conflict of interest.

\section{REFERENCES}

Alfawaz H, Alqedairi A, Al-Dahman YH, et al. (2019) Evaluation of root canal morphology of mandibular premolars in a Saudi population using cone beam computed tomography: A retrospective study. The Saudi dental journal 31(1): 137-142.

Awawdeh LA and Al-Qudah AA (2008) Root form and canal morphology of mandibular premolars in a Jordanian population. International endodontic journal 41(3): 240-248.

Azeem RA and Sureshbabu NM (2018) Clinical performance of direct versus indirect composite restorations in posterior teeth: A systematic review. Journal of conservative dentistry: JCD 21(1): 2-9.

Berman DDS L and Hargreaves KM (2015) Cohen's Pathways of the Pulp Expert Consult. Elsevier Health Sciences.

Çaliskan MK, Pehlivan Y, Sepetçioglu F, et al. (1995) Root canal morphology of human permanent teeth in a Turkish population. Journal of endodontics 21(4): 200-204.

Dou L, Li D, Xu T, et al. (2017) Root anatomy and canal morphology of mandibular first premolars in a Chinese 
population. Scientific reports 7(1): 750 .

Glassman GD (1987) Flare-up with associated paresthesia of a mandibular second premolar with three root canals. Oral surgery, oral medicine, and oral pathology 64(1): 110-113.

Govindaraju L, Neelakantan P and Gutmann JL (2017) Effect of root canal irrigating solutions on the compressive strength of tricalcium silicate cements. Clinical oral investigations 21(2): 567-571.

Gulabivala K, Aung TH, Alavi A, et al. (2001) Root and canal morphology of Burmese mandibular molars. International endodontic journal 34(5): 359-370.

Hajihassani N, Roohi N, Madadi K, et al. (2017) Evaluation of Root Canal Morphology of Mandibular First and Second Premolars Using Cone Beam Computed Tomography in a Defined Group of Dental Patients in Iran. Scientifica. DOI: 10.1155/2017/1504341.

Huang Y-D, Wu J, Sheu R-J, et al. (2015) Evaluation of the root and root canal systems of mandibular first premolars in northern Taiwanese patients using cone-beam computed tomography. Journal of the Formosan Medical Association = Taiwan yi zhi 114(11): 1129-1134.

Iyer VH, Indira R, Ramachandran S, et al. (2006) Anatomical variations of mandibular premolars in Chennai population. Indian journal of dental research: official publication of Indian Society for Dental Research 17(1): 7-10.

Janani K and Sandhya R (2019) A survey on skills for cone beam computed tomography interpretation among endodontists for endodontic treatment procedure. Indian journal of dental research: official publication of Indian Society for Dental Research 30(6): 834-838.

Jenarthanan S and Subbarao C (2018) Comparative evaluation of the efficacy of diclofenac sodium administered using different delivery routes in the management of endodontic pain: A randomized controlled clinical trial. Journal of conservative dentistry: JCD 21(3): 297-301.

Kacharaju KR, Hari P, Yee A, et al. (2019) Analysis of Mandibular Premolars Root Canal Morphology Using Radiographic and Cross-Sectional Techniques in Malaysian Population. Dental hypotheses 10(1). Medknow Publications and Media Pvt. Ltd.: 14.

Kazemipoor M, Hajighasemi A and Hakimian R (2015) Gender difference and root canal morphology in mandibular premolars: A cone-beam computed tomography study in an Iranian population. Contemporary clinical dentistry 6(3): 401-404.

Khandelwal A and Palanivelu A (2019) Correlation Between Dental Caries And Salivary Albumin In Adult Population In Chennai: An In Vivo Study. Brazilian Dental Science 22(2): 228-233.

Kottoor J, Albuquerque D, Velmurugan N, et al. (2013) Root anatomy and root canal configuration of human permanent mandibular premolars: a systematic review. Anatomy research international 2013: 254250.
Llena C, Fernandez J, Ortolani PS, et al. (2014) Conebeam computed tomography analysis of root and canal morphology of mandibular premolars in a Spanish population. Imaging science in dentistry 44(3): 221227.

Malli Sureshbabu N, Selvarasu K, V JK, et al. (2019) Concentrated Growth Factors as an Ingenious Biomaterial in Regeneration of Bony Defects after Periapical Surgery: A Report of Two Cases. Case reports in dentistry 2019: 7046203.

Manohar MP and Sharma S (2018) A survey of the knowledge, attitude, and awareness about the principal choice of intracanal medicaments among the general dental practitioners and nonendodontic specialists. Indian journal of dental research: official publication of Indian Society for Dental Research 29(6): 716-720. Martins JNR, Marques D, Francisco H, et al. (2018) Gender influence on the number of roots and root canal system configuration in human permanent teeth of a Portuguese subpopulation. Quintessence international 49(2): 103-111.

Miyoshi S, Fujiwara J, Yamamoto K, et al. (1974) Statistical analysis of bifurcated root canals in mandibular premolars. Japanese Journal of Oral Biology. DOI: 10.2330/joralbiosci1965.16.16.

Nandakumar M and Nasim I (2018) Comparative evaluation of grape seed and cranberry extracts in preventing enamel erosion: An optical emission spectrometric analysis. Journal of conservative dentistry: JCD 21(5): 516-520.

Neelakantan P, Subbarao C, Ahuja R, et al. (2010) Conebeam computed tomography study of root and canal morphology of maxillary first and second molars in an Indian population. Journal of endodontia 36(10): 1622-1627.

Patel S, Dawood A, Ford TP, et al. (2007) The potential applications of cone beam computed tomography in the management of endodontic problems. International endodontic journal 40(10): 818-830.

Poorni S, Srinivasan MR and Nivedhitha MS (2019) Probiotic strains in caries prevention: A systematic review. Journal of conservative dentistry: JCD 22(2): 123-128.

Rajakeerthi R and Ms N (2019) Natural Product as the Storage medium for an avulsed tooth - A Systematic Review. Cumhuriyet Dental Journal 22(2): 249-256. Rajendran R, Kunjusankaran RN, Sandhya R, et al. (2019) Comparative Evaluation of Remineralizing Potential of a Paste Containing Bioactive Glass and a Topical Cream Containing Casein Phosphopeptide-Amorphous Calcium Phosphate: An in Vitro Study. Pesquisa brasileira em odontopediatria e clinica integrada 19(1): 1-10.

Ramarao S and Sathyanarayanan U (2019) CRA Grid - A preliminary development and calibration of a paper-based objectivization of caries risk assessment in undergraduate dental education. Journal of conservative dentistry: JCD 22(2): 185-190. 
Serman NJ and Hasselgren G (1992) The radiographic incidence of multiple roots and canals in human mandibular premolars. International endodontic journal 25(5): 234-237.

Sert S and Bayirli GS (2004) Evaluation of the root canal configurations of the mandibular and maxillary permanent teeth by gender in the Turkish population. Journal of endodontia 30(6): 391-398.

Siddique R and Nivedhitha MS (2019) Effectiveness of rotary and reciprocating systems on microbial reduction: A systematic review. Journal of conservative dentistry: JCD 22(2): 114-122.

Siddique R, Sureshbabu NM, Somasundaram J, et al. (2019) Qualitative and quantitative analysis of precipitate formation following interaction of chlorhexidine with sodium hypochlorite, neem, and tulsi. Journal of conservative dentistry: JCD 22(1): 40-47.

Siddique R, Nivedhitha MS and Jacob B (2019) Quantitative analysis for detection of toxic elements in various irrigants, their combination (precipitate), and para-chloroaniline: An inductively coupled plasma mass spectrometry study. Journal of conservative dentistry: JCD 22(4): 344-350.

Suomalainen A, Kiljunen T, Käser Y, et al. (2009) Dosimetry and image quality of four dental cone beam computed tomography scanners compared with multislice computed tomography scanners. Dento maxillo facial radiology 38(6): 367-378.

Tachibana H and Matsumoto K (1990) Applicability of $\mathrm{X}$-ray computerized tomography in endodontics. Dental traumatology: official publication of International Association for Dental Traumatology 6(1). Wiley Online Library: 16-20.

Teja KV, Ramesh S and Priya V (2018) Regulation of matrix metalloproteinase-3 gene expression in inflammation: A molecular study. Journal of conservative dentistry: JCD 21(6): 592-596.

Tsiklakis K, Donta C, Gavala S, et al. (2005) Dose reduction in maxillofacial imaging using low dose Cone Beam CT. European journal of radiology 56(3): 413-417.

Velmurugan N, Kandaswamy D and Sandhya R (2010) Assessment of root canal morphology of mandibular first premolars in the Indian population using spiral computed tomography: An in vitro study. Indian Journal of Dental Research. DOI: 10.4103/0970-9290.66626.

Vertucci FJ (1978) Root canal morphology of mandibular premolars. Journal of the American Dental Association 97(1): 47-50.

Vertucci FJ (1984) Root canal anatomy of the human permanent teeth. Oral surgery, oral medicine, and oral pathology 58(5): 589-599.

Vertucci FJ (2005) Root canal morphology and its relationship to endodontic procedures. Endodontic Topics 10(1): 3-29.

Weine FS, Healey HJ, Gerstein H, et al. (1969) Canal configuration in the mesiobuccal root of the maxillary first molar and its endodontic significance. Oral Surgery, Oral Medicine, Oral Pathology. DOI: 10.1016/00304220(69)90237-0.

Yu X, Guo B, Li K-Z, et al. (2012) Cone-beam computed tomography study of root and canal morphology of mandibular premolars in a western Chinese population. BMC medical imaging 12: 18.

Zaatar EI, al-Kandari AM, Alhomaidah S, et al. (1997) Frequency of endodontic treatment in Kuwait: radiographic evaluation of 846 endodontically treated teeth. Journal of endodontia 23(7): 453-456. 